\title{
Radiative dynamical mass of planar charged fermion in a constant homogeneous magnetic field
}

\author{
V.R. Khalilov ${ }^{\mathrm{a}}$ \\ Faculty of Physics, M.V. Lomonosov Moscow State University, 119991 Moscow, Russia
}

Received: 29 November 2018 / Accepted: 22 February 2019 / Published online: 5 March 2019

(C) The Author(s) 2019

\begin{abstract}
The effective Lagrangian and mass operator are calculated for planar charged massive and massless fermions in a constant external homogeneous magnetic field in the one-loop approximation of the $2+1$ dimensional quantum electrodynamics $\left(\mathrm{QED}_{2+1}\right)$. We obtain the renormalizable effective Lagrangian and the fermion mass operator for a charged fermion of mass $m$ and then calculate these quantities for the massless case. The radiative corrections to the mass of charged massless fermion when it occupies the lowest Landau level are found for the cases of the pure $\mathrm{QED}_{2+1}$ as well as the so-called reduced $\mathrm{QED}_{3+1}$ on a 2-brane. The fermion masses were found can be generated dynamically in an external magnetic field in the pure $\mathrm{QED}_{2+1}$ if the charged fermion has small bare mass $m_{0}$ and in the reduced $\mathrm{QED}_{3+1}$ on a 2-brane even at $m_{0}=0$. The dynamical mass seems to be likely to be revealed in monolayer graphene in the presence of constant homogeneous magnetic field (normal to the graphene sample).
\end{abstract}

\section{Introduction}

Quantum systems of planar charged fermions in external electromagnetic fields are interesting in view of possible applications of the corresponding field-theory models to a number of condensed-matter quantum effects such as, for example, the quantum Hall effect [1] and high-temperature superconductivity [2] as well as in connection with problems of graphene (see, [3-6]). In graphene, the electron dynamics near the Fermi surface can be described by the Dirac equation in $2+1$ dimensions for a zero-mass charged fermion [4] though the case of massive charged fermions is also of interest [7]. The field-theory models applied for study are the pure $2+1$ dimensional quantum electrodynamics $\left(\mathrm{QED}_{2+1}\right)$ as well as the so-called reduced $\mathrm{QED}_{3+1}$ on a 2-brane. In the latter model, fermions are confined to a plane, neverthe-

a e-mail: khalilov@phys.msu.ru less the electromagnetic interaction between them is threedimensional $[8,9]$.

The radiative one-loop shift of an electron energy in the ground state in a constant homogeneous magnetic field in $\mathrm{QED}_{2+1}$ was calculated in [10] and the one-loop electron self-energy in the topologically massive $\mathrm{QED}_{2+1}$ at finite temperature and density was obtained in [11]. The effective Lagrangian, the electron mass operator and the density of vacuum electrons (induced by the background field) in an external constant homogeneous magnetic field were derived in the one-loop $\mathrm{QED}_{2+1}$ approximation in [12].

Since the effective fine structure constant in graphene is large, the $\mathrm{QED}_{2+1}$ effects can be significant already in the one-loop approximation. The polarization operator in graphene in a strong constant homogeneous magnetic field perpendicular to the graphene membrane has been obtained in the one-loop approximation of the $\mathrm{QED}_{2+1}$ in [9, 13-15]. The effective potential and vacuum current in graphene in a superposition of a constant homogeneous magnetic field and an Aharonov-Bohm vortex was studied in [16]. We also note that the induced vacuum current in the field of a solenoid perpendicular to the graphene sample was investigated in [17], and the vacuum polarization of massive and massless fermions in an Aharonov-Bohm vortex in the oneloop approximation of the $\mathrm{QED}_{2+1}$ was studied in [18]. The one-loop self-energy of a Dirac electron of mass $m$ in a thin medium simulating graphene in the presence of external magnetic field was investigated in the reduced $\mathrm{QED}_{3+1}$ on a 2brane in [19], in which it was shown that the radiative mass correction in the lowest Landau level does not vanish at the limit $m \rightarrow 0$.

In this work, we calculate the effective Lagrangian and the mass operator of planar charged fermions in the presence of an external constant homogeneous magnetic field in the one-loop approximation of the $\mathrm{QED}_{2+1}$. We also calculate the radiative corrections to the mass of charged fermion when it occupies the lowest Landau level for the cases of the 
pure $\mathrm{QED}_{2+1}$ as well as the so-called reduced $\mathrm{QED}_{3+1}$ on a 2-brane. The fermion masses were found can be generated dynamically in an external magnetic field in the pure $\mathrm{QED}_{2+1}$ if the charged fermion has small bare mass $m_{0}$ and in the reduced $\mathrm{QED}_{3+1}$ on a 2 -brane even at $m_{0}=0$. The dynamical mass seems to be likely to be revealed in monolayer graphene in the presence of constant homogeneous magnetic field (normal to the graphene sample) though we also see that an one-loop result is not accurate certainly when the coupling constant is large (see, [19]).

We shall adopt the units where $c=\hbar=1$.

\section{Eigenfunctions, the Green's function for the Dirac equation in a constant magnetic field in $2+1$ dimensions. Effective Lagrangian}

As far as the charged fermions are supposed to move in a plane the exact solutions and the Green's function for the Dirac equation in a constant homogeneous magnetic field can be found in $2+1$ dimensions. The Dirac equation for a fermion in an external electromagnetic field in $2+1$ dimensions is written just as in 3+1 dimensions nevertheless the Dirac $\gamma^{\mu}$ matrix algebra in $2+1$ dimensions is known to be represented in terms of the two-dimensional Pauli matrices $\sigma_{j}$

$\gamma^{0}=\sigma_{3}, \quad \gamma^{1}=i \zeta \sigma_{1}, \quad \gamma^{2}=i \sigma_{2}$,

where the parameter $\zeta= \pm 1$ can label two types of fermions in accordance with the signature of the two-dimensional Dirac matrices [20]; it can be applied to characterize two states of the fermion spin (spin "up" and "down") [21].

Then, the Dirac equation for a fermion of mass $m$ and charge $e=-e_{0}<0$ "minimally" interacting with the background electromagnetic field is written in the covariant form as

$\left(\gamma_{\mu} P^{\mu}-m\right) \Psi(x)=0$,

where $P^{\mu}=p^{\mu}-e A^{\mu} \equiv\left(P_{0}, P_{1}, P_{2}\right)$ is the generalized fermion momentum operator (a three-vector) and $p^{\mu}=\left(i \partial_{t},-i \partial_{x},-i \partial_{y}\right) \equiv\left(p_{0}, p_{1}, p_{2}\right)$. We take the vector potential in the Cartesian coordinates in the Landau gauge $A_{0}=0, A_{1}=0, A_{2}=B x$, then the magnetic field is defined as $B=\partial_{1} A_{2}-\partial_{2} A_{1} \equiv F_{21}$, where $F_{\mu v}$ is the electromagnetic field tensor.

The squared Dirac operator is

$(\gamma P)^{2}=P_{0}^{2}-P_{1}^{2}-P_{2}^{2}-e \sigma_{3} B$.

The matrix function $E_{p}$, introduced by Ritus [22] for the case of $3+1$ dimensions, satisfies the equation

$(\gamma P)^{2} E_{p}=p^{2} E_{p}$, where the eigenvalue $p^{2}$ can be any real number and in the magnetic field considered $E_{p}$ also is an eigenfunction of the operators

$$
\begin{aligned}
i \partial_{t} E_{p}= & p_{0} E_{p}, \quad-i \partial_{y} E_{p}=p_{2} E_{p}, \\
& \left(P_{1}^{2}+P_{2}^{2}-e \sigma_{3} B\right) E_{p}=2|e B| k E_{p}, \quad k=0,1 \ldots
\end{aligned}
$$

It is obvious that $p_{0}, p_{2}$ and $2|e B| n$ label the solutions of Dirac Eq. (2) as well as the $E_{p}$ eigenfunctions of operators (4) and (5) can also classifies by the eigenvalues $\zeta= \pm 1$ of $\sigma_{3}$. The eigenfunctions $E_{p \zeta}(t, \mathbf{r})$ are given by

$E_{p \zeta}(t, \mathbf{r})=\frac{1}{2^{3 / 2} \pi} e^{-i p_{0} t+i p_{2} y} U_{n}(X) w_{\zeta}$,

where the normalized functions $U_{n}(X)$ are expressed through the Hermite polynomials $H_{n}(X)$ as

$$
\begin{aligned}
U_{n}(X) & =\frac{|e B|^{1 / 4}}{\left(2^{n} n ! \pi^{1 / 2}\right)^{1 / 2}} e^{-X^{2} / 2} H_{n}(X), \\
X & =\sqrt{|e B|}\left(x-p_{2} / e B\right),
\end{aligned}
$$

$n=k+\operatorname{sign}(e B) \zeta / 2-1 / 2, \quad n=0,1, \ldots$, and $w_{\zeta}$ are eigenvectors of $\sigma_{3}$.

The eigenfunctions of the Dirac equation Hamiltonian

$H_{D}=\sigma_{1} P_{2}-\sigma_{2} P_{1}+\sigma_{3} m$

are

$$
\begin{aligned}
\Psi(t, \mathbf{r})= & \frac{1}{\sqrt{2 E_{n}}}\left(\begin{array}{l}
\sqrt{E_{n}+\zeta m} U_{n}(X) \\
-\operatorname{sign}(e B) \sqrt{E_{n}-\zeta m} U_{n-1}(X)
\end{array}\right) \\
& \times \exp \left(-i E_{n} t+i p_{2} y\right),
\end{aligned}
$$

where

$$
E_{n}=\sqrt{m^{2}+2 n|e B|}
$$

is the energy eigenvalues (the Landau levels). All the energy levels except the lowest level $(n=0)$ with $\zeta=1$ for $e B>0$ and $\zeta=-1$ for $e B<0$ are doubly degenerate on spin $\zeta= \pm 1$. This means that the eigenvalues of the fermion energy except the lowest level are actually spin-independent in the configuration under investigation. For definiteness, we consider the case where $e B<0$. It should be also emphasized that $\Psi(t, \mathbf{r})$ are not eigenfunctions of $\sigma_{3}$.

The electron propagator in an external constant homogeneous magnetic field in 3+1 dimensions was found for the first time by Schwinger [23]. In the $2+1$ dimensions it was obtained in [24] in the momentum representation (see, also, [15]) and in [12] in the coordinate (proper-time) representation in the form 


$$
\begin{aligned}
& S^{c}\left(t, t^{\prime}, \mathbf{r}, \mathbf{r}^{\prime}, B\right) \\
& =-\frac{i^{1 / 2}}{8 \pi^{3 / 2}} \int_{0}^{\infty} \frac{d z}{s^{1 / 2} \sin z} \exp \left[-i\left(m^{2}-i \epsilon\right)-i \frac{\left(t-t^{\prime}\right)^{2}}{4 s}\right. \\
& \left.+i z \frac{\left(x-x^{\prime}\right)^{2}+\left(y-y^{\prime}\right)^{2}}{4 s \tan z}+i e B \frac{\left(x-x^{\prime}\right)\left(y-y^{\prime}\right)}{2}\right] \\
& \quad \times\left[\left(\frac{\gamma^{0}\left(t-t^{\prime}\right)}{2 s}+m\right) \exp \left(i \zeta \sigma_{3} z\right)\right. \\
& \left.-z \frac{\left(\gamma^{1}\left(x-x^{\prime}\right)+\gamma^{2}\left(y-y^{\prime}\right)\right.}{2 s \sin z}\right],
\end{aligned}
$$

where $z=|e B| s$ and $s$ is the "proper time".

The effective action $V(B)=\int d^{3} x L_{e f f}(B)$, where $L_{e f f}(B)$ is the effective Lagrangian and $x^{\mu}=x^{0}=t, x^{1}=$ $x, x^{2}=y$, is determined the causal Green's function $S^{c}$ as follows

$V(B)=i \operatorname{Tr} \log S^{c}=i \int d^{3} x \operatorname{tr}\left(x\left|\log S^{c}\right| x\right)$,

and from Eq. (11) we derive

$L_{e f f}(B)=\frac{(-i)^{1 / 2}}{8 \pi^{3 / 2}} \int_{0}^{\infty} \frac{d s}{s^{3 / 2}} e^{-i m^{2} s}\left(|e B| \cot |e B| s-\frac{1}{s}\right)$

Rotating the integration contour by $-\pi / 2$, we have

$L_{e f f}(B)=-\frac{|e B|^{3 / 2}}{8 \pi^{3 / 2}} \int_{0}^{\infty} \frac{d z}{z^{3 / 2}} e^{-m^{2} z /|e B|}\left(\operatorname{coth} z-\frac{1}{z}\right)$

and integrating (14), one obtains (see, [12])

$L_{e f f}(B)=-\frac{(e B)^{2}}{24 m \pi}\left(1-\frac{(e B)^{2}}{20 m^{4}}\right), \quad|e B| \ll m^{2}$

and

$L_{e f f}(B) \approx \frac{(e B)^{2}}{12 m \pi^{3 / 2}}, \quad|e B| \gg m^{2}$.

In Eq. (15) the first term can be interpreted as the magnetic energy of the vacuum [25]. In the $\mathrm{QED}_{3+1}$ the nonrenormalized effective Lagrangian involves a similar $B^{2}$ term with a logarithmic factor whose argument depends on the cutoff parameter. This term is then combined with the $B^{2}$ term in the initial Lagrangian $L_{0}\left(B^{2}\right)$, which implies fermion-charge and external magnetic-field renormalizations.

For massless case it is convenient to write the one-loop correction $L_{1}$ to the Lagrangian density $L_{0}=-B^{2} / 4 \pi$ for the magnetic field as

$L_{1}=\frac{|e B|}{2 \pi} \sum_{n=1}^{\infty}\left|E_{n}^{\prime}\right|$

where the factor $|e B| / 2 \pi$ takes into account quantum degeneracy of Landau levels per unit surface area, $E_{n}^{\prime}=\sqrt{2|e B| n}$ and the zero modes are to be omitted (see, [26,27]). A massless fermion does not have a spin degree of freedom in $2+1$ dimensions [28] but the Dirac equation for charged massless fermions in an external magnetic field in $2+1$ dimensions keeps the spin parameter. Therefore, all the energy levels except the lowest level $n=0$ are also doubly degenerate. This sum is divergent. Using for $\left|E_{n}^{\prime}\right|$ the Fock-Schwinger proper-time representation in the form [29]

$\sqrt{2|e B| n}=-(\pi)^{-1 / 2} \int_{0}^{\infty} \frac{d s}{s^{1 / 2}} \frac{d}{d s} e^{-2|e B| n s}$

and applying for (17) the zeta-function regularization [26,27, 30], after some calculations one can bring Eq. (17) formally to the form

$$
\begin{aligned}
L_{1} & =-\frac{(e B)^{3 / 2}}{2 \sqrt{2} \pi^{3 / 2}} \int_{0}^{\infty} \frac{x^{-3 / 2}}{e^{x}-1} d x \\
& \equiv-\frac{(e B)^{3 / 2}}{2 \sqrt{2} \pi^{3 / 2}} \Gamma(-1 / 2) \zeta(-1 / 2),
\end{aligned}
$$

where $\Gamma(z)$ and $\zeta(z)$ are respectively the gamma and $\zeta$ functions. Then using the Riemann identity [31]

$\zeta(z)=\Gamma(1-z) 2^{z} \pi^{z-1} \cos [\pi(1-z) / 2] \zeta(1-z)$,

we obtain the renormalizable effective Lagrangian for charged massless fermions in the final form :

$L_{1}=-\frac{(e B)^{3 / 2}}{4 \sqrt{2} \pi^{2}} \zeta(3 / 2)$.

This formula coincides with the corresponding result obtained in $[26,27]$.

\section{Mass operator of a charged fermion in a constant homogeneous magnetic field}

In the coordinate representation, the mass operator of a charged massive fermion in the considered external field in $2+1$ dimensions is given by

$$
\begin{aligned}
M\left(x, x^{\prime}\right)= & i e^{2} \gamma^{\mu} S^{c}\left(x, x^{\prime}\right) \gamma^{\nu} S_{\mu \nu}\left(x-x^{\prime}\right) \\
& -i e^{2} \gamma^{\mu} S_{\mu \nu}\left(x-x^{\prime}\right) \operatorname{tr}\left(\gamma^{\nu} S^{c}\left(x, x^{\prime}\right)\right),
\end{aligned}
$$

where $S_{\mu \nu}\left(x-x^{\prime}\right)$ is the photon propagation function. We note that in the $\mathrm{QED}_{3+1}$, the second term in the right-hand side of Eq. (22) is absent in the constant homogeneous electromagnetic field because the induced vacuum electric current vanishes. In the pure $\mathrm{QED}_{2+1}$, the Chern-Simons field dynamically generated in the effective Heisenberg-Euler Lagrangian by the background magnetic field contributes in this second term in the model under consideration. The second term in the mass operator of a charged fermion in a constant homogeneous magnetic field $M\left(x, x^{\prime}\right)$ violates the spatial and temporal invariance and therefore cannot be observed, even in principle. We shall not consider this term. 
The effect due to the Chern-Simons field can possibly be observed in a condensed-matter model with a finite density of fermions as the density of charged fermions induced by the background magnetic field.

We shall use the usual photon propagator in $2+1$ dimensions (in the Feynman gauge)

$\frac{1}{k^{2}-i \epsilon}=i \int_{0}^{\infty} d u e^{-i\left(k^{2}-i \epsilon\right)}$,

as well as the "effective" internal photon propagator for the reduced $\mathrm{QED}_{3+1}$ on a 2-brane in which the photon is allowed to also propagate in the "bulk" [8]

$\frac{1}{\sqrt{k^{2}-i \epsilon}}=\sqrt{\frac{i}{\pi}} \int_{0}^{\infty} d u \frac{e^{-i\left(k^{2}-i \epsilon\right)}}{\sqrt{u}}$,

In the coordinate (proper-time) representation the photon propagator in $2+1$ dimensions reads as

$$
\begin{aligned}
& S_{\mu \nu}\left(t-t^{\prime}, \mathbf{r}-\mathbf{r}^{\prime}\right)=g_{\mu \nu} \frac{i^{1 / 2}}{8 \pi^{3 / 2}} \int_{0}^{\infty} \frac{d u}{u^{3 / 2}} \\
& \quad \times \exp \left[-\epsilon u+i \frac{\left(t-t^{\prime}\right)^{2}-\left(x-x^{\prime}\right)^{2}-\left(y-y^{\prime}\right)^{2}}{4 u}\right] .
\end{aligned}
$$

The internal photon propagator for the reduced $\mathrm{QED}_{3+1}$ on a 2-brane is given by Eq. (25) in which $i^{1 / 2} / 8 \pi^{3 / 2}$ and $u^{-3 / 2}$ are replaced by $i / 8 \pi^{1 / 2}$ and $u^{-2}$, respectively.

We determine the mass operator in the $E_{p}$ representation as

$M\left(p, p^{\prime}, B\right)=\int d^{3} z \int d^{3} z^{\prime} \bar{E}_{p}(z) M\left(z, z^{\prime}, B\right) E_{p}^{\prime}\left(z^{\prime}\right)$,

where $\bar{E}_{p}=\gamma^{0} E_{p}^{\dagger} \gamma^{0}$ and $E_{p}^{\dagger}$ is the Hermitian conjugate matrix function. The mass operator is diagonal in the $E_{p}$ representation

$M\left(p, p^{\prime}, B\right)=\delta^{3}\left(p-p^{\prime}\right) M(p, B)$.

We now calculate the mass operator in the special reference frame with using the fermion propagator (11), the photon propagator (25) and $E_{p}$-matrix function (6). It is convenient to introduce variables $t_{-}=t-t^{\prime}, x_{-}=x-x^{\prime}, y_{-}=$ $y-y^{\prime}, t_{+}=\left(t+t^{\prime}\right) / 2, x_{+}=\left(x+x^{\prime}\right) / 2, y_{+}=\left(y+y^{\prime}\right) / 2$. It is seen that the $t_{+}$and $y_{+}$integrations give $2 \pi \delta\left(p_{0}-p_{0}^{\prime}\right)$ and $2 \pi \delta\left(p_{2}-p_{2}^{\prime}\right)$. We then use the arising $\delta$-functions, integrate with respect to $t_{-}$and obtain in the terms independent of $t_{-}$ the factor

$2 \sqrt{\frac{\pi s u}{i(s+u)}} \exp \left(\frac{i p_{0}^{2} s u}{s+u}\right)$.

The first-order terms in $t_{-}$then contain an extra factor $2 p_{0} s u /(s+u)$.
We integrate over $x_{+}$with applying formula

$$
\begin{aligned}
& \int_{-\infty}^{\infty} \sqrt{e B} \exp \left(-i y_{-} x\right) U_{n}(\eta) U_{k}\left(\eta^{\prime}\right) d x \\
& \quad=\exp [i(n-k)) v] I_{n k}(z),
\end{aligned}
$$

where $U_{l}(\eta)$ is given by Eq. (7), $\eta=\sqrt{e B}\left(x+x_{-}\right), \quad \eta^{\prime}=$ $\sqrt{e B}\left(x-x_{-}\right), \quad v=\arctan \left(y_{-} / x_{-}\right)$, and $I_{n k}(z)$ is the Laguerre function in $z=|e B|\left(x_{-}^{2}+y_{-}^{2}\right) / 2$, related to the Laguerre polynomial

$L_{s}^{l}(z)=e^{z} z^{-l} \frac{d^{s}}{d z^{s}}\left(z^{s+l} e^{-z}\right)$

as

$I_{n k}(z)=\frac{1}{\sqrt{n ! k !}} e^{-z / 2} z^{(n-k) / 2} L_{k}^{n-k}(z)$.

The integration over $x_{-}$and $y_{-}$is performed in polar coordinates with using the formulas

$$
\begin{aligned}
\int_{0}^{2 \pi} d \phi e^{i(n-k) \phi} & =2 \pi \delta_{n k}, \phi=\arctan \left(y_{-} / x_{-}\right), \\
\int_{0}^{\infty} d z e^{-a z} L_{n}^{0}(z) & =\frac{(a-1)^{n}}{a^{n+1}}, \operatorname{Re}(a)>0 .
\end{aligned}
$$

After these integrations the mass operator (27) at $s u /(s+$ $u) \rightarrow 0$ has the ultraviolet divergency of the vacuum origin and needs in the renormalization. The renormalized mass operator in an external electromagnetic field can be represented in the form

$$
\begin{aligned}
M_{r}(p, B)= & M(p, B)-M^{0}+M^{0}-\left(M^{0}\right)_{\gamma p=m} \\
& -\left(\frac{\partial M^{0}}{\partial \gamma p}\right)_{\gamma p=m}(\gamma p-m) \equiv M_{c}(p, B)+M_{r}^{0},
\end{aligned}
$$

where $M^{0}=M(p, B=0)$, the expression $M_{c}(p, B)=$ $M(p, B)-M^{0}$ does not contain the divergences and $M_{r}^{0}$ is the regularized mass operator of fermion in the vacuum, which vanishes on the mass shell.

As a result of this renormalization, we obtain the renormalized mass operator with the photon propagator (25) in the form

$$
\begin{aligned}
& M_{c}(p, B) \\
& =-e^{2} \frac{i^{3 / 2}}{8 \pi^{3 / 2}} \int_{0}^{\infty} d s \int_{0}^{\infty} d u\left[\frac{|e B|}{\Delta(s+u)^{1 / 2} \sin (|e B| s)}\right. \\
& \exp \left[-i s m^{2}+i p_{0}^{2} s u /(s+u)-2 i n \phi\right] \\
& \quad \times\left[\left(3 M I-\gamma^{0} P_{0}\right) e^{-i \phi}-\gamma^{2} P_{2}\right] \\
& \left.\quad-\frac{\exp \left[-i s m^{2}+i p_{0}^{2} s u /(s+u)\right]}{(s+u)^{3 / 2}}\left[3 m I-\sigma_{3} \frac{p_{0} u}{s+u}\right]\right],
\end{aligned}
$$


where $\Delta=\sqrt{(e B u)^{2}+(1+e B u \cot (e B s))^{2}}, \phi=\arctan$ $[e B u /(1+e B u \cot (e B s))], I$ is the unit two-column matrix, and

$$
\begin{aligned}
M i & =\frac{p_{0} u}{s+u} \sin (|e B| s)+m \cos (|e B| s), \\
P_{0} & =\frac{p_{0} u}{s+u} \cos (|e B| s)+i m \sin (|e B| s), \\
P_{2} & =\sqrt{2|e B| n} \frac{e B u}{\Delta \sin (|e B| s)} .
\end{aligned}
$$

Putting $s^{\prime}=|e B| s, \quad u^{\prime}=|e B| u$, we obtain

$$
\begin{aligned}
& M_{c}(p, B)=-e^{2} \frac{i^{3 / 2}}{8 \pi^{3 / 2} \sqrt{|e B|}} \int_{0}^{\infty} d s^{\prime} \\
& \int_{0}^{\infty} d u^{\prime} e^{\left[-i s^{\prime} m^{2} /|e B|+i p_{0}^{2} s^{\prime} u^{\prime} /|e B|\left(s^{\prime}+u^{\prime}\right)\right]} \\
& \times\left[\left(\left(3 M I-\sigma_{3} P_{0}\right) \exp [-i(2 n+1) \phi]\right.\right. \\
& \left.-i \sigma_{2} \frac{\sqrt{2|e B| n} u^{\prime} \exp [-i 2 n \phi]}{\Delta^{\prime}}\right) \frac{1}{\Delta^{\prime} \sqrt{s^{\prime}+u^{\prime}}}- \\
& \left.-\left[3 m I-\sigma_{3} \frac{p_{0} u^{\prime}}{s^{\prime}+u^{\prime}}\right] \frac{1}{\left(s^{\prime}+u^{\prime}\right)^{3 / 2}}\right],
\end{aligned}
$$

where $\Delta^{\prime}=\sqrt{u^{\prime 2}+2 u^{\prime} \sin s^{\prime} \cos s^{\prime}+\sin ^{2} s^{\prime}}, \quad \phi=\arctan$ $\left[u^{\prime} /\left(1+u^{\prime} \cot \left(s^{\prime}\right)\right)\right]$.

It is worth while noting that the fermion propagator (i.e. the fermion Green's function) in a constant homogeneous magnetic field in $2+1$ dimensions (11) is derived with taking account of all Landau levels.

Now, let us perform a known change of variables (see [32], p. 44, Eq. (3.12) and, also, [19]) $s^{\prime}=s v, \quad u^{\prime}=s(1-v)$, and introduce a variable $z=i s v^{2}$, which, in fact, is equivalent to the turning of integration path over $s$ on $\pi / 2$ (a Wick rotation). We shall calculate the mass correction for the (on mass-shell) level $p_{0}=m, \quad n=0$. As a result of all these transformations, the mass operator can be written as

$$
\begin{aligned}
& M_{c}(B)=\frac{e^{2} m}{8 \pi^{3 / 2}} \int_{0}^{\infty} d z \sqrt{z} e^{-(z / l)} \int_{0}^{1} \frac{d v}{v} \\
& \times\left[\left(\begin{array}{ll}
4-2 v+2 v \exp [-2 z / v] & 0 \\
0 & 8-4 v+2 v \exp [-2 z / v]
\end{array}\right)\right. \\
& \left.\times \frac{1}{2 z(1-v)+v^{2}(1-\exp [-2 z / v])}-\left(\begin{array}{ll}
2+v & 0 \\
0 & 4-v
\end{array}\right) \frac{1}{z}\right]
\end{aligned}
$$

where $l=|e B| / m^{2}$.

In order to determine the mass correction of a charged fermion $\delta m$ in the lowest Landau level $n=0, \zeta=-1$, we must calculate the matrix element of the self-energy operator (35) between two states with $n=0, \zeta=-1$, defined by Eq. (9). After simple calculations, we obtain

$$
\begin{aligned}
\delta m= & \frac{e^{2} m}{8 \pi^{3 / 2}} \int_{0}^{\infty} d z \sqrt{z} e^{-(z / l)} \int_{0}^{1} \frac{d v}{v} \\
& {\left[\frac{8-4 v+2 v \exp [-2 z / v]}{2 z(1-v)+v^{2}(1-\exp [-2 z / v])}-\frac{4-v}{z}\right] . }
\end{aligned}
$$

The mass correction of a charged fermion $\delta m_{r}$ in the level $n=0, \zeta=-1$ for the reduced $\mathrm{QED}_{3+1}$ on a 2-brane is given by

$$
\begin{aligned}
\delta m_{r}= & \frac{e^{2} m}{8 \pi} \int_{0}^{\infty} d z e^{-(z / l)} \int_{0}^{1} \frac{d v}{\sqrt{1-v}} \\
& {\left[\frac{8-4 v+2 v \exp [-2 z / v]}{2 z(1-v)+v^{2}(1-\exp [-2 z / v])}-\frac{4-v}{z}\right] . }
\end{aligned}
$$

It is well to note that Eqs. (35), (36) and (37) are taken into account the contributions of the virtual electrons occupying all Landau levels, but also when obtaining Eq. (35) from Eq. (34) we took account of that the needed diagonal matrix element of operator (34) must be calculated for the lowest state with $n=0, \zeta=-1$.

It is useful the integration over $z$ in the limits $[0, \infty)$ to divide into two regions $\left[0, z_{0}\right]$ plus $\left[z_{0}, \infty\right.$ ) [33] (see, also, [19]) with $z_{0} \sim 1$. Then, when integrating into region $\left[z_{0}, \infty\right)$ the exponentials can be neglected and integral between the limits 0 and $z_{0}$ is small compared with that in the limits $\left[z_{0}, \infty\right)$ and it can be neglected. Having made the integrations over $v$ we obtained

$\delta m=\frac{e^{2} m}{8 \pi^{3 / 2}} \int_{z_{0}}^{\infty} d z e^{-(z / l)}\left(\frac{4}{\sqrt{z}}+\frac{2 \ln (2 z)}{\sqrt{z}}\right)$

and

$\delta m_{r}=\frac{e^{2} m}{8 \pi} \int_{z_{0}}^{\infty} d z e^{-(z / l)}\left(\frac{2 \sqrt{2} \pi}{\sqrt{z}}-\frac{20}{3 z}\right)$.

It should be emphasized that though the first term in integrand of (39) has the form $2 \sqrt{2} \pi / \sqrt{z}$ at $z \gg 1$ the exact calculation of corresponding integral over $v$ at $z=1$ gives $2 \sqrt{2} \pi$.

Finally, integrating over $z$ we find the mass corrections in the form

$$
\begin{aligned}
\delta m= & \frac{e^{2}}{4 \pi} \sqrt{|e B|}\left[\left(\ln \left(\frac{|e B|}{m^{2}}\right)+2+\ln 2\right) \operatorname{erfc}\left(\frac{1}{\sqrt{l}}\right)\right. \\
& -2 \ln 2-\mathcal{C}]
\end{aligned}
$$

and

$\delta m_{r}=\frac{e^{2}}{4 \pi} \sqrt{|e B|}\left[\sqrt{2} \pi^{3 / 2} \operatorname{erfc}\left(\frac{1}{\sqrt{l}}\right)-\frac{10}{3 \sqrt{l}} \Gamma\left(0, \frac{1}{l}\right)\right]$, 
where (see, for example, [35])

$\operatorname{erfc}(z)=\frac{2}{\sqrt{\pi}} \int_{z}^{\infty} e^{-t^{2}} d t, \quad \Gamma(a, z)=\int_{z}^{\infty} e^{-t} t^{a-1} d t$

and we put $z_{0}=1$. In the limit $l \rightarrow \infty$

$\operatorname{erfc}\left(\frac{1}{\sqrt{l}}\right) \approx 1-\frac{2}{\sqrt{\pi l}}, \quad \Gamma\left(0, \frac{1}{l}\right) \approx \ln l-\mathcal{C}$.

Here $\mathcal{C}=0.57721$ is the Euler constant [36].

At the limit $m \rightarrow 0$, we obtain

$\delta m=\frac{e^{2}}{4 \pi} \sqrt{|e B|}\left[\ln \left(\frac{|e B|}{m_{0}^{2}}\right)+2-\ln 2-\mathcal{C}\right], \quad m_{0} \rightarrow 0$

and

$\delta m_{r}=\frac{e^{2}}{2 \sqrt{2}} \sqrt{\pi|e B|}$.

Equation (45) coincides with Eq. (63) obtained in [19].

In order to estimate the magnitude of the magnetic field strength which can be of interest for application to graphene, we recover the units $c$ and $\hbar$ and rewrite Eq. (45) as follows

$\delta m_{r} c^{2}=\frac{\alpha \sqrt{\pi}}{2 \sqrt{2}} \sqrt{|e B| \hbar c}, \quad \alpha=\frac{e^{2}}{\hbar c}$.

The numerical estimation is obtained in the form

$\delta m_{r} c^{2} \approx 0.5 \cdot 10^{-4} \alpha \sqrt{|B|} e V$.

If the constant $\alpha$ is considered as "effective fine structure constant" for graphene then $\alpha \sim 1$, and as it follows from the Eq. (47) the mass correction $\delta m_{r} c^{2} \approx 1 e V$ in the magnetic field of the strength $B=4 T\left(4 \cdot 10^{4} G\right)$. Of course, there is a question about the validity of one-loop result when the corresponding constant of expansion can be of order 1 but the study of higher orders lie beyond the scope of given work.

It should be noted that Eqs. (44) and (45) describe the main terms of radiative mass corrections in massive $\mathrm{QED}_{2+1}$ and reduced $\mathrm{QED}_{3+1}$ on a 2-brane, respectively, in the limits $|e B| \gg m^{2}$. We see these corrections differ, in principle, from the mass correction in standard massive $\mathrm{QED}_{3+1}$ in the limits $|e B| \gg m^{2}$ (see, $[33,37]$ ), in which the leading term is determined by

$\delta m_{3+1}=\frac{e^{2}}{4 \pi} m \ln ^{2}\left(\frac{|e B|}{m^{2}}\right)$.

Equations (44) and (45) show that, in the presence of external constant homogeneous magnetic field, the fermion mass can be generated dynamically in $\mathrm{QED}_{2+1}$ at small bare mass $m_{0}$ and in reduced $\mathrm{QED}_{3+1}$ on a 2-brane at $m_{0}=0$. Thus, the latter model, in a constant homogeneous magnetic field, cannot stay massless in the one-loop approximation [19].

\section{Resume}

In this work we calculated the effective Lagrangian and the mass operator of planar charged fermions in an external constant homogeneous magnetic field in the one-loop approximation of the $\mathrm{QED}_{2+1}$. We also calculated the mass corrections of charged fermion in the lowest Landau level. The fermion mass can be generated dynamically in a constant homogeneous magnetic field in the pure $\mathrm{QED}_{2+1}$ if the charged fermion has small bare mass $m_{0}$ and in the reduced $\mathrm{QED}_{3+1}$ on a 2-brane even at $m_{0}=0$. We believe the mass generation to be due to renormalization and to closely resemble to the dimensional transmutation phenomenon which occurs in massless relativistic field theories [38]. In our case the cutoff parameter, in fact, depending upon $e B$ transmutes in an arbitrary mass. The dynamical mass seems to be likely to be revealed in monolayer graphene in the presence of constant homogeneous magnetic field (normal to the graphene sample).

Data Availability Statement This manuscript has no associated data or the data will not be deposited. [Authors' comment: This is a theoretical study and no experimental data has been listed.]

Open Access This article is distributed under the terms of the Creative Commons Attribution 4.0 International License (http://creativecomm ons.org/licenses/by/4.0/), which permits unrestricted use, distribution, and reproduction in any medium, provided you give appropriate credit to the original author(s) and the source, provide a link to the Creative Commons license, and indicate if changes were made. Funded by SCOAP $^{3}$.

\section{References}

1. R.E. Prange, S.M. Girvin (eds.), The Quantum Hall Effect, 4th edn. (Springer, New York, 1990)

2. F. Wilczek, Fractional Statistics and Anyon Superconductivity (World Scientific, Singapore, 1990)

3. K.S. Novoselov et al., Science 306, 666 (2004)

4. A.H. Castro Neto et al., Rev. Mod. Phys. 81, 109 (2009)

5. N.M.R. Peres, Rev. Mod. Phys. 82, 2673 (2010)

6. V.N. Kotov et al., Rev. Mod. Phys. 84, 1067 (2012)

7. F. Guinea, M.I. Katsnelson, A.K. Geim, Nat. Phys. 6, 30 (2009)

8. E.V. Gorbar, V.P. Gusynin, V.A. Miransky, Phys. Rev. D 64, 105028 (2001)

9. E.V. Gorbar, V.P. Gusynin, V.A. Miransky, I.A. Shovkovy, Phys. Rev. B 66, 045108 (2002)

10. I.M. Ternov, A.V. Borisov, K.V. Zhukovsky, Moscow Univ. Phys. Bull. Phys. Astron. 1, 71 (1997)

11. K.V. Zhukovsky, P.A. Eminov, Phys. Lett. B 359, 155 (1995)

12. V.R. Khalilov, Theor. Math. Phys. 121, 1606 (1999)

13. V.P. Gusynin, S.G. Sharapov, Phys. Rev. B 73, 245411 (2006)

14. P.K. Pyatkovskiy, V.P. Gusynin, Phys. Rev. B 83, 075422 (2011)

15. V.R. Khalilov, I.V. Mamsurov, Eur. Phys. J. C 75(4), 167 (2015)

16. E.A. Stepanov, VCh. Zhukovsky, Phys. Rev. B 94, 094101 (2016)

17. R. Jackiw, A.I. Milstein, S.-Y. Pi, I.S. Terekhov, Phys. Rev. B 80, 033413 (2009)

18. V.R. Khalilov, Eur. Phys. J. C 74, 2708 (2014) 
19. B. Machet, Non-vanishing at $m \rightarrow 0$ of the 1-loop self-mass of an electron of mass $m$ propagating in a graphene-like medium in a constant external magnetic field, e-print arXiv:1607.00838v1 [hep$\mathrm{ph}]$ (2016)

20. Y. Hosotani, Phys. Lett. B 319, 332 (1993)

21. C.R. Hagen, Phys. Rev. Lett. 64, 503 (1990)

22. V.I. Ritus, Trudy Fiz. Inst. Lebedev 111, 5 (1979)

23. J. Schwinger, Phys. Rev. 82, 664 (1951)

24. V.P. Gusynin, V.A. Miransky, I.A. Shovkovy, Phys. Rev. D 52, 4718 (1995)

25. A. Neagu, A.M.J. Schakel, Phys. Rev. D 48, 1785 (1993)

26. M.I. Katsnelson, G.E. Volovik, M.A. Zubkov, Ann. Phys 331, 160 (2013)

27. M.I. Katsnelson, G.E. Volovik, M.A. Zubkov, Euler - Heisenberg effective action and magnetoelectric effect in multilayer graphene, e-print arXiv:1206.3973v3 [cond-mat.mes-hall/] (24 Dec 2012)
28. R. Jackiw, V.P. Nair, Phys. Rev. D 43, 1933 (1991)

29. R.J. Hughes, Phys. Lett. B 148, 215 (1984)

30. S.W. Hawking, Commun. Math. Phys. 55, 133 (1977)

31. H. Bateman, A. Erdelyi, Higher Transcendental Functions (McGraw-Hill, New York, 1953)

32. W. Dittrich, M. Reuter, Effective Lagrangians in Quantum Electrodynamics, (Springer). Lecture Notes in Physics 220, 44 (1985)

33. B. Jancovici, Phys. Rev. 187, 2275 (1969)

34. B. Machet, Int. J. Mod. Phys. A 31, 1650071 (2016)

35. F.W.J. Olwer, Introduction to Asymptotics and Special Functions (Academic Press, New York, 1974)

36. I.S. Gradshteyn, I.M. Ryzhik, Table of Integrals, Series, and Products, 5th edn. (Academic Press, San Diego, 1994)

37. I.M. Ternov, V.G. Bagrov, O.F. Dorofeev, V.G. Bordovitsin, ZhETF 55, 2273 (1968)

38. S. Coleman, E. Weinberg, Phys. Rev. D 7, 1888 (1973) 